\title{
SNARER: New Molecular Descriptors for SNARE Proteins Classification
}

\author{
Alessia Auriemma Citarella ( $\nabla$ aauriemmacitarella@unisa.it ) \\ University of Salerno \\ Luigi Di Biasi \\ University of Salerno \\ Michele Risi \\ University of Salerno \\ Genoveffa Tortora \\ University of Salerno
}

\section{Research Article}

Keywords: SNARE, Protein Classification, Machine Learning, Random Forest, AdaBoost, KNN

Posted Date: July 29th, 2021

DOl: https://doi.org/10.21203/rs.3.rs-710235/v1

License: (c) (i) This work is licensed under a Creative Commons Attribution 4.0 International License. Read Full License 


\title{
SNARER: new molecular descriptors for SNARE proteins classification
}

\author{
Alessia Auriemma Citarella ${ }^{*}$, Luigi Di Biasi, Michele Risi and Genoveffa Tortora
}

\author{
${ }^{*}$ Correspondence: \\ aauriemmacitarella@unisa.it \\ Department of Computer Science, \\ University of Salerno, Fisciano, IT \\ Full list of author information is \\ available at the end of the article
}

\begin{abstract}
Background: SNARE proteins play an important role in different biological functions. This study aims to investigate the contribution of a new class of molecular descriptors (called SNARER) related to the chemical-physical properties of proteins in order to evaluate the performance of binary classifiers for SNARE proteins.

Results: We constructed a SNARE proteins balanced dataset, D128, and an unbalanced one, DUNI, on which we tested and compared the performance of the new descriptors presented here in combination with the feature sets (GAAC, CTDT, CKSAAP and 188D) already present in the literature. The machine learning algorithms used were Random Forest, k-Nearest Neighbors and AdaBoost and oversampling and subsampling techniques were applied to the unbalanced dataset. The addition of the SNARER descriptors increases the precision for all considered ML algorithms. In particular, on the unbalanced DUNI dataset the accuracy increases in parallel with the increase in sensitivity while on the balanced dataset D128 the accuracy increases compared to the counterpart without the addition of SNARER descriptors, with a strong improvement in specificity. Our best result is the combination of our descriptors SNARER with CKSAAP feature on the dataset D128 with $92.3 \%$ of accuracy, $90.1 \%$ for sensitivity and $95 \%$ for specificity with the RF algorithm.

Conclusions: The performed analysis has shown how the introduction of molecular descriptors linked to the chemical-physical and structural characteristics of the proteins can improve the classification performance. Additionally, it was pointed out that performance can change based on using a balanced or unbalanced dataset. The balanced nature of training can significantly improve forecast accuracy.
\end{abstract}

Keywords: SNARE; Protein Classification; Machine Learning; Random Forest; AdaBoost; KNN

\section{Background}

SNARE (Soluble N-ethylmaleimide sensitive factor Attachment protein Receptor) is a protein superfamily involved in the molecular trafficking between the different cellular compartments [1]. This protein family includes members from yeasts to mammalian cells, evolutionarily conserved. Vesicle-mediated transport is essential for basic cellular processes, such as the secretion of proteins and hormones, the release of neurotransmitters, the phagocytosis of pathogens by the immune system and the transport of molecules from one compartment of the cell to another.Vesicular transport involves membrane receptors responsible for the vescicles recognition, the activation of the membrane fusion and reorganization and the consequent release of 
the vesicular content in the extracellular space (exocytosis) or inside the cell (endocytosis). Specifically, SNARE complexes mediate membrane fusion during diffusion processes, providing bridging bond between SNARE proteins associated with both membranes [2].

SNARE proteins consist of motifs of 60-70 amino acids containing hydrophobic heptad repeats which form coiled-coil structures. The core of the SNARE complex is represented by $4 \alpha$ helix bundle, as evidenced by the available crystallographic structures [3]. The center of the bundle contains 16 stacked layers which are all hydrophobic, except the central layer "0", which is called ionic layer and which contains 3 highly conserved glutamines (Q) and a conserved arginine (R) residue (see Figure 1).

SNARE proteins were initially divided into two categories: vesicle or v-SNARE, which are incorporated into the vesicle membranes, and target or t-SNARE, which are associated with the target membranes. A more recent subdivision is based on their structural characteristics by dividing them into R-SNARE and Q-SNARE. The R-SNARE proteins contain an arginine residue $(\mathrm{R})$ which contributes to the formation of the complex while Q-SNARE proteins contain a glutamine residue (Q) and, according to their position in the bundle of four helices, they are classified in turn as $Q_{a}, Q_{b}$ or $Q_{c}$ [4].

In recent years, attention to SNARE proteins has increased due to scientific studies which have shown the implication of SNAREs in some neural disorders for their crucial role in the neuronal and neurosensory release at the level of synaptic endings [5]. The neurotransmitters release is a temporally and spatially regulated process and it occurs thousands of times per minute. In this context, SNARE complexes are continuously subject to tightly regulated assembly and disassembly. Impairment at any stage of this release can lead to hypo or hyperactivity of neurotransmitter release causing dysfunctions which compromise the balance of synaptic communication. There are evidences that these substances seem to be involved in the course of neurodegenerative diseases (such as Alzheimer and Parkinson), in neurodevelopment (autism) and in psychiatric disorders (such as bipolar disorder and schizophrenia as well as depression). Different studies have shown the involvement of mutated or not properly regulated SNARE genes in the development of these disorders $[6,7,8,9,10,11]$.

Nowadays the protein sequences collection is constantly growing. There is a need to have efficient classification systems able to define the functionality of a protein

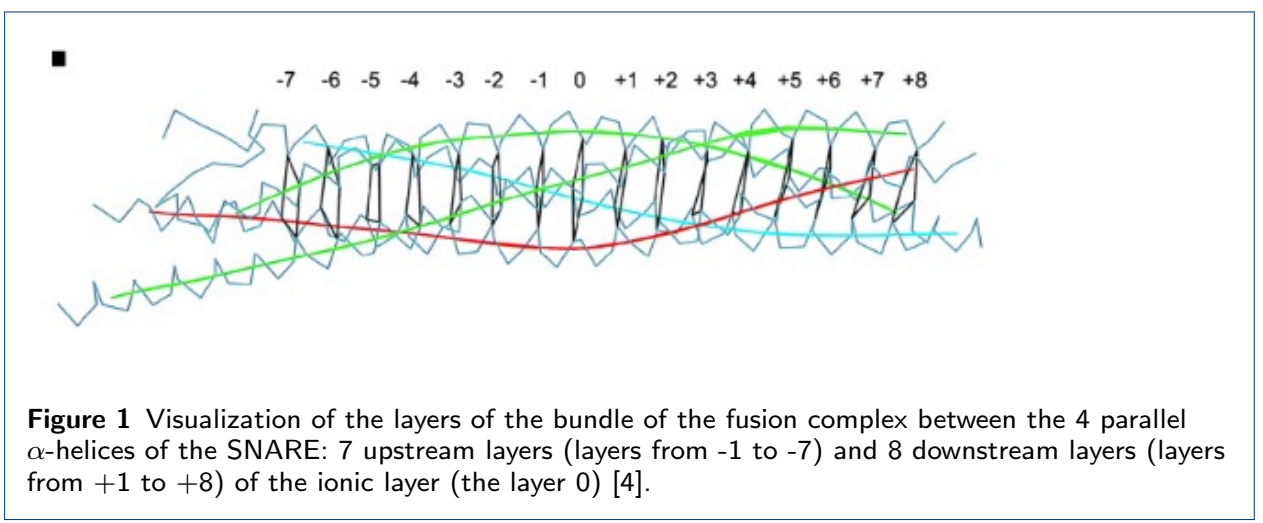


based on its chemical-physical properties and to label the sequence with greater precision. The more information we can gather about a certain protein, the better our ability to fit it into a more complex biological framework. This is evident and useful especially when considering a protein with an initially unknown function. The most used approach consists in evaluating whether there are functional motifs and domains in the protein which allow to characterize it starting from its amino acid sequence and evaluating its belonging to a protein family in which the members have similar three-dimensional structures, similar functions and significant sequence similarities. Knowledge of the protein family representatives is therefore necessary to define their role and their mechanisms in a specific physiological and pathological biological path. High-throughput sequencing techniques generate lots of big data belonging to different biological domains, including protein sequencing [12]. These huge amounts of data (up to petabytes) must be computationally analyzed with ever newer techniques for the identification of different genomic and protein regions. The current challenge is to contribute to this post-sequencing analysis and classification and to ensure greater precision in the available protein sequences discrimination.

The importance of the evolutionary SNAREs super-family is strictly connected to their role in different cellular functions and different pathological conditions [13, 14], which push researchers to deepen their recognition in the biological pathways.

\section{Related Works}

Since SNARE proteins are involved in numerous biological processes, studies have slightly increased in recent decades in order to identify and classify these proteins but the papers dealing with this topic are still few. In the literature there are documents that are based on different techniques, ranging from statistical models to the use of convolutional neural networks.

Kloepper et al. [15] have implemented a web-based interface which allows the new sequences submission to the Hidden Markov Models (HMM) for the four main groups of the SNARE family, in order to classify SNARE proteins based on sequence alignment and reconstruction of the phylogenetic tree. For their study, a set of $\sim 150$ SNARE proteins is used in conjunction with the highly conserved motif which is the sequence pattern signature representing the family of SNARE proteins. For SNARE proteins, this motif is an extended segment arranged in heptad repeats, a structural motif consisting of a seven-amino-acid repeating pattern. The extraction of HMM profiles, which allow to identify evolutionary changes in a set of correlated sequences, returns information on the occupancy and position-specific frequency of each amino acid in the alignment. Using this method, the authors are able to obtain a classification accuracy of at least $95 \%$ for nineteen of the twenty HMM profiles generated and to perform a cluster analysis based on functional subgroups.

Nguyen et al. [16] have disclosed a model with two-dimensional convolutional network and position-specific scoring matrix profiles for the SNARE proteins identification. The authors used multiple hidden layers for their models, in particular 2D sub-layers such as zero padding, convolutional, max pooling and fully-connected layers with different number of filters. Their model achieves a sensitivity of $76.6 \%$, an accuracy of $89.7 \%$ and a specificity of $93.5 \%$.

More recently, in 2020, Guilin Li [17] has proposed an hybrid model which combines the random forest algorithm with the oversampling filter and 188D feature 
extraction method. His work proposes different combinations of feature extraction methods, filtering methods and classification algorithms such as KNN, RF and AdaBoost for the classification of SNARE proteins. Since those results are shown only graphically, it is not possible to have a clear comparison with our results.

\section{Methods}

Dataset preparation

We have constructed two datasets, respectively named DUNI and D128. Both datasets were used for the evaluation of each classifier's robustness in unbalanced and balanced training environment, in order to avoid learning bias into classification training. In both datasets, SNARE proteins were downloaded from UNIPROT ${ }^{[1]}$. For this purpose, we selected all the proteins with molecular function "SNAP receptor activity", identified with the unique GENE Ontology [18] alphanumeric code GO: 0005484. The dataset DUNI consists of 276 SNAREs and 806 non-SNAREs. On this unbalanced dataset, we applied the subsampling and ovesampling techniques used in [17]. The balanced dataset D128 is composed of 64 SNARE from UNIPROT and 64 non-SNARE protein sequences downloaded from the PDB database ${ }^{[2]}$.

In order to create a balanced and non-redundant dataset and improve the dataset quality, all SNARE protein sequences in FASTA format have been processed with the CD-HIT (Cluster Database at High Identity with Tolerance) ${ }^{[3]}$ program which returns a set of non-redundant representative sequences in output. CD-HIT uses an incremental clustering algorithm. In the first analysis, it sorts the sequences in length descending order and creates the first cluster in which the longest sequence is the representative one. Then the sequences are compared with the clusters representatives. If the similarity with a representative is above a certain threshold, the sequence will be grouped in that cluster. Alternatively, a new cluster is created with that sequence as the representative [19]. The similarity threshold chosen was $25 \%$. This step is very important, since it allows the removal of sequences which exceed the similarity threshold and that could invalidate the analysis causing unwanted bias. Sequence similarity is measured by the similar residues percentage between two sequences. The lower the sequence similarity, the greater the likelihood of having representative proteins in the dataset which consequently show no redundancy [20].

\section{Feature extraction methods}

In order to analyze the data deriving from protein sequences with ML techniques, a numerical representation is required for each amino acid in the protein. For this reason, a series of numerical parameters are often used which act as chemicalphysical and structural descriptors of proteins. The combination of a different set of carefully chosen descriptors increases classification efficiency and allows predicting functional protein families [21].

So there are some feature extraction methods commonly used in machine learning. Identifying the right features for machine learning-based protein classification is one of the open issues in this field. The right features combination is important to ensure greater classifier model accuracy [22].

\footnotetext{
${ }^{[1]}$ https://www. uniprot.org/

${ }^{[2]}$ https : //www.rcsb.org/

${ }^{[3]}$ http://weizhongli-lab.org/cd-hit/
} 
In the literature, over the years, many indices and features of amino acids have been identified for classification methods, such as amino acid composition (AAC), auto-correlation functions [23] or pseudo amino acid composition (PseAAC) [24].

We chose the following four descriptors to compare our SNARER descriptors with those currently used in the SNARE proteins classification.

- GAAC (Grouped amino acid composition) groups the 20 amino acids into five groups based on their chemical-physical properties and calculates the frequency for each of the five groups in a protein sequence. Specifically, the five groups are the following: positive charge $(K, R, H)$, negative charge (D, E), aromatic group (F, Y, W), aliphatic group (A, G, I, L, M, V) and uncharge $(\mathrm{C}, \mathrm{N}, \mathrm{P}, \mathrm{Q}, \mathrm{S}, \mathrm{T})$.

- CTDT (Composition/Transition/Distribution) represents the amino acid composition patterns distribution of a specific chemical-physical or structural property in the protein sequence. The final $\mathrm{T}$ represents the transition between three types of patterns (neutral group, hydrophobic group and polar group) of which the percentage of occurrence frequency is calculated [25].

- CKSAAP are sequence-based features which, given a sequence, count all adjacent amino acid pairs, considering k-spaced amino acid pairs. Since there are 20 amino acids, for each value of $\mathrm{k}$ (from 0 to 5) there are 400 possible pairs of amino acids, for a total of 2400 features [26].

- 188D features constitute a features vector of which the first 20 represent the frequencies of each amino acid while eight types of chemical-physical properties (such as hydrophobicity, polarizability, polarity, surface tension, etc) allow us to calculate the remaining 168 features. In fact, for each type of property 21 features are extracted [27].

For our purpose, we have selected 24 descriptors, 19 of which come from AAindex, i.e., the Amino Acid index database [28], on the basis of the chemical-physical, electrical and energy charge characteristics of the SNARE proteins. They are listed in Table 1. The others (i.e., Steric parameter, polarizability, Volume, Isoelectric point, Helix probability, Sheet probability and Hydrophobicity) are the amino acid parameter sets defined by Fauchere et al. [29].

\section{Classification algorithms}

Following [17], given the high reported performances, we used the same three classification algorithms in order to evaluate how accuracy varies with the SNARER descriptors utilization. Thus, we have compared three different ML algorithms: AdaBoost (ADA) K-Nearest Neighbor classifier (KNN) and Random Forest (RF).

- AdaBoost is a machine learning meta-algorithm used in binary classification. AdaBoost is an adaptive algorithm which generates a model that is overall better than the single weak classifiers, adapting to the weak hypothesis accuracy and generating one weighted majority hypothesis in which the weight of each weak hypothesis is a function dependent of its accuracy. At each iteration, a new weak classifier is sequentially added which corrects its predecessor until a final hypothesis with a low relative error is found [30].

- KNN is a supervised learning algorithm used for predictive classification and regression problems. The basis of the operation of this algorithm is to classify 
Table 1 The SNARER descriptors.

\begin{tabular}{lll}
\hline Code & Description & Source \\
\hline ARGP820102 & Signal sequence helical potential\% & \\
CHAM830101 & The Chou-Fasman parameter of the coil conformation & \\
CHAM830107 & A parameter of charge transfer capability & \\
CHAM830108 & A parameter of charge transfer donor capability & \\
CHOP780204 & Normalized frequency of N-terminal helix & \\
CHOP780205 & Normalized frequency of C-terminal helix & \\
EISD860101 & Solvation free energy & AAindex \\
FAUJ880108 & Localized electrical effect & \\
FAUJ880111 & Positive charge & \\
FAUJ880112 & Negative charge & \\
GUYH850101 & Partition energy & \\
JANJ780101 & Average accessible surface area & \\
KRIW790101 & Side chain interaction parameter & \\
ZIMJ680102 & Bulkiness & \\
ONEK900102 & Helix formation parameters (delta delta G) & \\
& Steric parameter & Fauchere et al. [29] \\
& Polarizability & \\
& Volume & \\
& Isoelectric point & \\
& Helix probability & \\
& Sheet probability & \\
& Hydrophobicity &
\end{tabular}

an object based on the similarity between the data, generally calculated by means of the Euclidean distance. In this way the space is partitioned into regions according to the learning objects similarity. This algorithm identifies a collection of $\mathrm{k}$ objects in the training set that are the most similar to the test object. So, a parameter k, chosen arbitrarily, allows us to identify the number of nearest neighbors, considering the $\mathrm{k}$ minimum distances. The prevalence of a certain class in this neighborhood becomes a forecast in order to assign a label to the object [31].

- $\mathrm{RF}$ is a supervised learning algorithm that combines many decision trees into one model by aggregation through bagging. The final result of the RF is represented by the class returned by the largest number of decision trees. In particular, the random forest algorithm learns from a random sample of data and trains on random characteristics subsets by splitting the nodes in each tree [32].

\section{Training and validation sessions}

All training sessions were conducted with Weka ML Platform (Waikato Environment for Knowledge Analysis), a software environment written in Java which allows the application of machine learning and data mining algorithms [33]. In order to speed-up analysis, an ad-hoc grid, based on the map/reduce paradigm, were used in order to distribute the work across multiple slaves [34]. Both data sets were used as the input for the training step for AdaBoost, KNN e RF classifiers. There were only two possible output classes: SNARE/ NON SNARE. Then, for each training session, we used the following cross-validation values: the range between 10 to 100 for k-fold and between 20 to $80 \%$ for hold out. Moreover, in addition to other parameters configured as in [17], we set $\mathrm{k}=1$ and Euclidean distance for the distanceFunction of $\mathrm{KNN}$; for the AdaBoost algorithm, default values are weightThreshold $=100$ and numIterations $=10$, whilst for RF numIterations $=100$.

The complete working set was composed of four logical parts: i) DUNI nonfiltered; ii) DUNI oversampled; iii) DUNI subsampled; iv) D128 non-filtered. For 
each training session, we generated $10 \mathrm{k}$-fold variants and 7 hold out variants. Then, for each variant we computed 100 training sessions of each of the three classifiers for each of the four descriptors. Thus, we distributed up to 836.000 training sessions among the distributed computing environment.

\section{Performance measurement}

We evaluated the ML models (Random Forest, AdaBoost and KNN) on the unbalanced dataset DUNI and on the balanced dataset D128. In order to estimate the prediction performance of the three ML algorithms, accuracy (ACC), sensitivity (SN) and specificity (SP) were used. The chosen metrics are described in the equations below:

$$
\begin{gathered}
\text { Accuracy }=\frac{T P+T N}{T N+F P+F N+T P} \\
\text { Sensitivity }=\frac{T P}{T P+F N} \\
\text { Specificity }=\frac{T N}{T N+F P}
\end{gathered}
$$

where TP, TN, FP and FN represent the number of true positives, true negatives, false positives and false negatives, respectively. Sensitivity is the percentage of positive entities correctly identified. Specificity measures the proportion of negative entities that are correctly identified.

In a biological sense, having a TP in our experiment means finding that a protein cataloged as a SNARE is recognized by the classifier as a SNARE.

The feature extraction methods were initially evaluated separately (GAAC, CKSAAP, CTDT and 188D) on the datasets D128 and DUNI, and subsequently these methods were extended with the SNARER descriptors addition disclosed in this work, here identified as extended classes "ext".

\section{Results and Discussion}

We used the SNARER descriptors and the three chosen ML algorithms on the unbalanced dataset DUNI and on the balanced dataset D128.

We have first considered four feature sets (GAAC, CTDT, CSKAAP and 188D) separately and then each one in combination with the SNARER descriptors class, identified with ".ext". The classification performances were evaluated with three metrics: average accuracy (ACC), average sensibility (SN) and average specificity (SP).

Results on the unbalanced dataset DUNI

Below, we have reported the experimental results conducted on the DUNI dataset. Related to the four protein feature extraction methods GAAC, CTDT, CKSAAP and $188 \mathrm{D}$, the average ACCs for the ML algorithms are included in a range between 
Table 2 Performance of average ACC on the DUNI dataset.

\begin{tabular}{cccc}
\hline & \multicolumn{3}{c}{ Accuracy } \\
\hline & RF & KNN & ADA \\
\hline GAAC & $76.1 \%$ & $85.1 \%$ & $77.9 \%$ \\
GAAC.ext & $\mathbf{9 0 . 4} \%$ & $\mathbf{9 1} \%$ & $\mathbf{8 6 . 1} \%$ \\
\hline CTDT & $76.1 \%$ & $83.1 \%$ & $76.7 \%$ \\
CTDT.ext & $\mathbf{9 1 . 5} \%$ & $\mathbf{9 2 . 6} \%$ & $\mathbf{8 1 . 6} \%$ \\
\hline CKSAAP & $91.1 \%$ & $90.04 \%$ & $83.7 \%$ \\
CKSAAP.ext & $\mathbf{9 1 . 7} \%$ & $90.02 \%$ & $\mathbf{8 7 . 4} \%$ \\
\hline 188D & $93.9 \%$ & $94.8 \%$ & $88.1 \%$ \\
188D.ext & $\mathbf{9 4 . 1} \%$ & $\mathbf{9 4 . 9} \%$ & $\mathbf{8 8 . 7} \%$ \\
\hline
\end{tabular}

$76 \%$ and $94.9 \%$. In Figure 2, histograms are used for the graphical comparison of the three ML techniques.

As shown in Table 2, the introduction of the SNARER class brings a strong improvement in combination with all the considered protein feature extraction methods. Overall, the best average accuracy is achieved with the KNN model and with the $188 \mathrm{D}$ feature set and the SNARER class combination. This combined model achieves also the best average SP while the best average SN is obtained with the RF model trained using both GAAC and CTDT features separately (see Table 3).

For RF, SN decreases imperceptibly in the extended classes with the new descriptors, remaining unchanged for the CKSAAP method. In contrast, for RF, SP increases with the extended classes, notably especially for the GAAC and CTDT extraction methods.

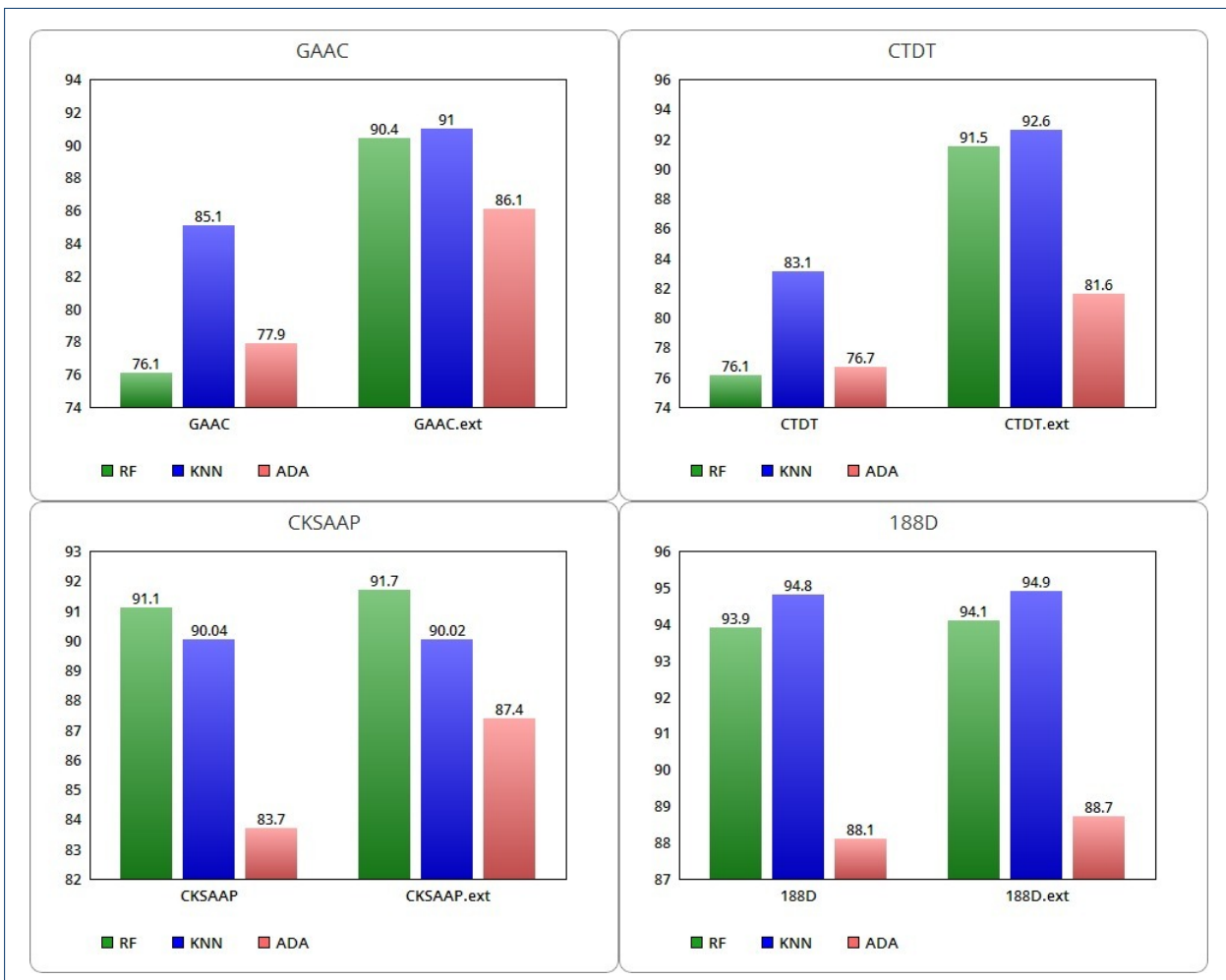

Figure 2 Comparison between GAAC, CTDT, CKSAAP and 188 D ACC with related extended classes with SNARER (on DUNI dataset). 
Table 3 Performance for average SN and SP on the DUNI dataset.

\begin{tabular}{ccccccc}
\hline & \multicolumn{3}{c}{ Sensitivity } & \multicolumn{3}{c}{ Specificity } \\
\hline & RF & KNN & ADA & RF & KNN & ADA \\
\hline GAAC & $\mathbf{9 9 . 8} \%$ & $90.3 \%$ & $83.6 \%$ & $7 \%$ & $7 \%$ & $\mathbf{6 1} \%$ \\
GAAC.ext & $\mathbf{9 7 . 2 \%}$ & $\mathbf{9 4 . 5} \%$ & $\mathbf{9 4 . 8} \%$ & $\mathbf{7 0 . 7} \%$ & $\mathbf{8 0 . 6} \%$ & $60.6 \%$ \\
\hline CTDT & $\mathbf{9 9 . 8} \%$ & $89.1 \%$ & $83.3 \%$ & $7.1 \%$ & $65.6 \%$ & $\mathbf{5 7 . 6} \%$ \\
CTDT.ext & $96.6 \%$ & $\mathbf{9 4 . 6} \%$ & $\mathbf{9 1 . 1} \%$ & $\mathbf{7 6 . 4} \%$ & $\mathbf{8 7} \%$ & $54 \%$ \\
\hline CKSAAP & $97.8 \%$ & $98 \%$ & $89.9 \%$ & $71.7 \%$ & $66.7 \%$ & $65.5 \%$ \\
CKSAAP.ext & $97.8 \%$ & $98 \%$ & $\mathbf{9 2} \%$ & $\mathbf{7 4} \%$ & $66.7 \%$ & $\mathbf{7 4} \%$ \\
\hline 188D & $\mathbf{9 7} \%$ & $\mathbf{9 6 . 6} \%$ & $\mathbf{9 2} \%$ & $85 \%$ & $89.5 \%$ & $76.7 \%$ \\
188D.ext & $\mathbf{9 6 . 8} \%$ & $96.5 \%$ & $\mathbf{9 2 . 4} \%$ & $\mathbf{8 6 . 3} \%$ & $\mathbf{9 0 . 1} \%$ & $\mathbf{7 8} \%$
\end{tabular}

The SN of KNN increases significantly in the extended classes referred to GAAC and CTDT and remains substantially unchanged for CKSAAP and 188D. The same trend is also shown for the SP of KNN, with a slight improvement of the extended $188 \mathrm{D}$ class. For the AdaBoost algorithm, we observe an increase in SN, mostly for the extended GAAC and CTDT classes, which however show a decrease in SP. The SP ADA, instead, increases for the extended classes CKSAAP and 188D. Overall, on the unbalanced dataset the use of extended classes with our SNARER descriptors results in an improvement in accuracy for the GAAC, CTDT, CKSAAP and 188D classes of all three ML models, except for KNN trained with CKSAAP. All selected ML algorithms achieve SN greater than $83 \%$, with the best SN of $99.8 \%$ RF achieved by GAAC and CTDT without extension.

By introducing the SNARER class for all four feature sets, the SN settles in a range between $91.1 \%$ of the ADA algorithm with the CTDT class and $98 \%$ of the KNN algorithm with the extended CKSAAP class. Regarding the SP, without the SNARER's descriptor extension, the range extends from a minimum of $7 \%$ of $\mathrm{RF}$ and KNN algorithms for the GAAC class to a maximum of $89.5 \%$ of KNN trained with the 188D feature set. With the SNARER class addition, an SN of $54 \%$ of ADA with CTDT feature set is obtained at a maximum of $90.1 \%$ of KNN trained on the dataset with $188 \mathrm{D}$ feature set. More specifically, the KNN model using the 188D extended class with SNARER descriptors, achieves better performance in all metrics except for SN, where the RF model trained with the GAAC features obtains the highest value.

In conclusion, on the unbalanced DUNI dataset, the new SNARER descriptors class guarantees an improvement in terms of ACC in combination with all four tested features sets and a clear improvement of SN and SP of some ML tested algorithms.

Results on the unbalanced dataset DUNI with oversampling and with subsampling Because the dataset DUNI is unbalanced, we have adopted subsampling and oversampling techniques.

With the oversampling technique on the DUNI dataset, the SNARER class produces a strong improvement in accuracy, more for the extended GAAC and CTDT classes for the three ML models RF, KNN and ADA, while the contribution to the CKSAPP and 188D feature sets remains substantially unchanged (as shown in Table 4). The same behavior is common to the average SN and average SP calculated for RF, KNN and ADA (see Table 5). Applying the subsampling technique to the DUNI dataset, we observe the same trend for SN but with a slight decrease, around 
Table 4 Performance of the average ACC on the DUNI dataset with oversampling and subsampling.

\begin{tabular}{ccccccc}
\hline & \multicolumn{3}{c}{ Oversampling } & \multicolumn{3}{c}{ Subsampling } \\
\hline & RF & KNN & ADA & RF & KNN & ADA \\
\hline GAAC & $94.7 \%$ & $96.3 \%$ & $73.12 \%$ & $75.2 \%$ & $79.2 \%$ & $72.6 \%$ \\
GAAC.ext & $\mathbf{9 8 . 0 3 \%}$ & $\mathbf{9 8 . 4 4 \%}$ & $\mathbf{8 5 . 0 2 \%}$ & $\mathbf{9 1 . 8 \%}$ & $\mathbf{8 6 . 4 \%}$ & $\mathbf{8 2 . 6 \%}$ \\
\hline CTDT & $93.9 \%$ & $96.1 \%$ & $70.4 \%$ & $74.6 \%$ & $78.1 \%$ & $71.7 \%$ \\
CTDT.ext & $\mathbf{9 8 \%}$ & $\mathbf{9 8 \%}$ & $\mathbf{8 6 . 3 \%}$ & $\mathbf{9 0 . 6 \%}$ & $\mathbf{8 9 . 7 \%}$ & $\mathbf{8 6 . 4 \%}$ \\
\hline CKSAAP & $\mathbf{9 9 . 0 7 \%}$ & $\mathbf{9 8 . 6 7 \%}$ & $84 \%$ & $\mathbf{9 3 . 1 \%}$ & $\mathbf{8 4 . 4 \%}$ & $83.5 \%$ \\
CKSAAP.ext & $99.01 \%$ & $98.6 \%$ & $\mathbf{8 9 . 1 \%}$ & $79 \%$ & $84.2 \%$ & $\mathbf{8 7 . 3 \%}$ \\
\hline 188D & $98.5 \%$ & $98.90 \%$ & $89.5 \%$ & $93.1 \%$ & $\mathbf{9 5 \%}$ & $86.6 \%$ \\
188D.ext & $98.5 \%$ & $\mathbf{9 8 . 9 5 \%}$ & $\mathbf{8 9 . 6 \%}$ & $\mathbf{9 3 . 5 \%}$ & $94 \%$ & $\mathbf{8 9 . 3 \%}$
\end{tabular}

Table 5 Performance for average SN and SP on the DUNI dataset with oversampling.

\begin{tabular}{ccccccc}
\hline & \multicolumn{3}{c}{ Sensitivity } & \multicolumn{3}{c}{ Specificity } \\
\hline RF & KNN & ADA & RF & KNN & ADA \\
\hline GAAC & $91.9 \%$ & $95 \%$ & $74.9 \%$ & $97.5 \%$ & $97.6 \%$ & $71.3 \%$ \\
GAAC.ext & $\mathbf{9 6 . 6 \%}$ & $\mathbf{9 7 . 8 \%}$ & $\mathbf{8 8 . 4 \%}$ & $\mathbf{9 9 . 4 \%}$ & $\mathbf{9 9 . 1 \%}$ & $\mathbf{8 1 . 6 \%}$ \\
\hline CTDT & $88.9 \%$ & $94 \%$ & 68.8 & $98.8 \%$ & $98.2 \%$ & $72 \%$ \\
CTDT.ext & $\mathbf{9 6 . 4 \%}$ & $\mathbf{9 6 . 9 \%}$ & $\mathbf{7 8 . 4 \%}$ & $\mathbf{9 9 . 5 \%}$ & $\mathbf{9 9 . 2 \%}$ & $\mathbf{9 4 . 3 \%}$ \\
\hline CKSAAP & $\mathbf{9 9 \%}$ & $\mathbf{9 9 . 2 \%}$ & $80.8 \%$ & $99.2 \%$ & $98.2 \%$ & $87.2 \%$ \\
CKSAAP.ext & $98.7 \%$ & $99.1 \%$ & $\mathbf{8 6 . 2 \%}$ & $\mathbf{9 9 . 3 \%}$ & $98.2 \%$ & $\mathbf{9 2 \%}$ \\
\hline 188D & $97.5 \%$ & $98.3 \%$ & $\mathbf{9 0 . 2 \%}$ & $\mathbf{9 9 . 7 \%}$ & $99.5 \%$ & $88.8 \%$ \\
188D.ext & $\mathbf{9 7 . 7 \%}$ & $98.3 \%$ & $89.4 \%$ & $99.3 \%$ & $\mathbf{9 9 . 7 \%}$ & $\mathbf{8 9 . 9 \%}$
\end{tabular}

Table 6 Performance for average SN and SP on the DUNI dataset with subsampling.

\begin{tabular}{ccccccc}
\hline & \multicolumn{3}{c}{ Sensitivity } & \multicolumn{3}{c}{ Specificity } \\
\hline & RF & KNN & ADA & RF & KNN & ADA \\
\hline GAAC & $75.7 \%$ & $76.1 \%$ & $73.6 \%$ & $74.6 \%$ & $82.2 \%$ & $71.7 \%$ \\
GAAC.ext & $\mathbf{8 8 . 8 \%}$ & $\mathbf{8 5 . 5 \%}$ & $\mathbf{8 0 . 8 \%}$ & $\mathbf{9 4 . 9 \%}$ & $\mathbf{8 7 . 3 \%}$ & $\mathbf{8 4 . 4 \%}$ \\
\hline CTDT & $78.3 \%$ & $76.4 \%$ & $73.9 \%$ & $71 \%$ & $79.7 \%$ & $69.6 \%$ \\
CTDT.ext & $\mathbf{8 6 . 6 \%}$ & $\mathbf{8 8 . 4 \%}$ & $\mathbf{8 1 . 9 \%}$ & $\mathbf{9 4 . 6 \%}$ & $\mathbf{9 0 . 9 \%}$ & $\mathbf{9 0 . 9 \%}$ \\
\hline CKSAAP & $\mathbf{9 0 . 9 \%}$ & $\mathbf{9 8 . 6 \%}$ & $83.3 \%$ & $\mathbf{9 5 . 3 \%}$ & $70.3 \%$ & $83.7 \%$ \\
CKSAAP.ext & $76.4 \%$ & $98.2 \%$ & $\mathbf{8 3 . 7 \%}$ & $81.5 \%$ & $70.3 \%$ & $\mathbf{9 0 . 9 \%}$ \\
\hline 188D & $90.9 \%$ & $\mathbf{9 5 . 3 \%}$ & $88 \%$ & $\mathbf{9 5 . 3} \%$ & $94.6 \%$ & $85.1 \%$ \\
188D.ext & $\mathbf{9 2 \%}$ & $93.1 \%$ & $\mathbf{8 8 . 8 \%}$ & $94.9 \%$ & $\mathbf{9 4 . 9 \%}$ & $\mathbf{8 9 . 9 \%}$
\end{tabular}

$2 \%-4 \%$, of the values when considering the extended classes CKSAAP and 188D. The same decrease value is also present for the average SPs of the same classes (see Table 6).

Results on the balanced dataset D128

Below we present the obtained classification results on the balanced dataset D128, with and without the addition of the SNARER descriptors. Table 7 reports the average accuracy performances of the ML algorithms without considering the SNARER descriptors in the balanced D128 dataset. In addition, histograms are depicted graphically in Figure 3: RF varies from a minimum of $71.1 \%$ with the use of the GAAC class to a maximum of $95.4 \%$ with the $188 \mathrm{D}$ class; KNN settles between a minimum of $64.2 \%$ with the use of GAAC to a maximum of $90 \%$ with the $188 \mathrm{D}$

Table 7 Performance of average ACC for the D128 dataset.

\begin{tabular}{cccc}
\hline & \multicolumn{3}{c}{ Accuracy } \\
\hline & RF & KNN & ADA \\
\hline GAAC & $71.1 \%$ & $64.2 \%$ & $70 \%$ \\
GAAC.ext & $\mathbf{8 4} \%$ & $\mathbf{6 5 . 4} \%$ & $\mathbf{8 4} \%$ \\
\hline CTDT & $73.4 \%$ & $66.4 \%$ & $70.3 \%$ \\
CTDT.ext & $\mathbf{8 8} \%$ & $\mathbf{6 8 . 7} \%$ & $\mathbf{8 4 . 1} \%$ \\
\hline CKSAAP & $92.2 \%$ & $72.4 \%$ & $80.7 \%$ \\
CKSAAP.ext & $\mathbf{9 2 . 3} \%$ & $\mathbf{7 4 . 1} \%$ & $\mathbf{8 9 . 4} \%$ \\
\hline 188D & $\mathbf{9 5 . 4} \%$ & $\mathbf{9 0} \%$ & $\mathbf{9 0 . 2} \%$ \\
188D.ext & $95.3 \%$ & $88.6 \%$ & $90 \%$
\end{tabular}


class; ADA varies from a minimum of $70 \%$ with GAAC to a maximum of $90.2 \%$ trained on the 188D class. Extended classes with SNARER descriptors shift these average ACC rates. In particular, RF varies from a minimum of $84 \%$ using the extension with GAAC to a maximum of $95.3 \%$ with the $188 \mathrm{D}$ class. KNN starts from a minimum of $65.4 \%$ with the extended GAAC class and reaches a maximum of $88.6 \%$ with the extended $188 \mathrm{D}$ class. ADA varies in a range between $84 \%$ with the GAAC.ext class to a maximum of $90 \%$ with the combined class $188 \mathrm{D}$.

By comparing the evaluated average ACCs, the SNARER class addition improves the classification performance in relation to the GAAC, CKSAAP and CTDT feature extraction methods while there is a slight decrease in the average ACCs for the 188D feature extraction class. Further analysis should be conducted to understand the reason for this decrease. In particular, the best classification results are obtained with the RF algorithm.

With the extended feature extraction methods, we can note that for the RF algorithm SN increases with GAAC and CTDT while it remains fundamentally unchanged for the other two descriptor classes (see Table 8). Also SP increases showing the same behavior. For the KNN algorithm, SN decreases for the GAAC and CTDT classes by $3 \%$ and $1 \%$ for the $188 \mathrm{D}$ class while it increases by $2 \%$ for the CKSAAP class. The SP of KNN instead increases for all classes except 188D, with a decrease of about $2 \%$. ADA improves in terms of SN on all extended classes while it decreases in SP by $0.8 \%$ when applied on the extended class $188 \mathrm{D}$.

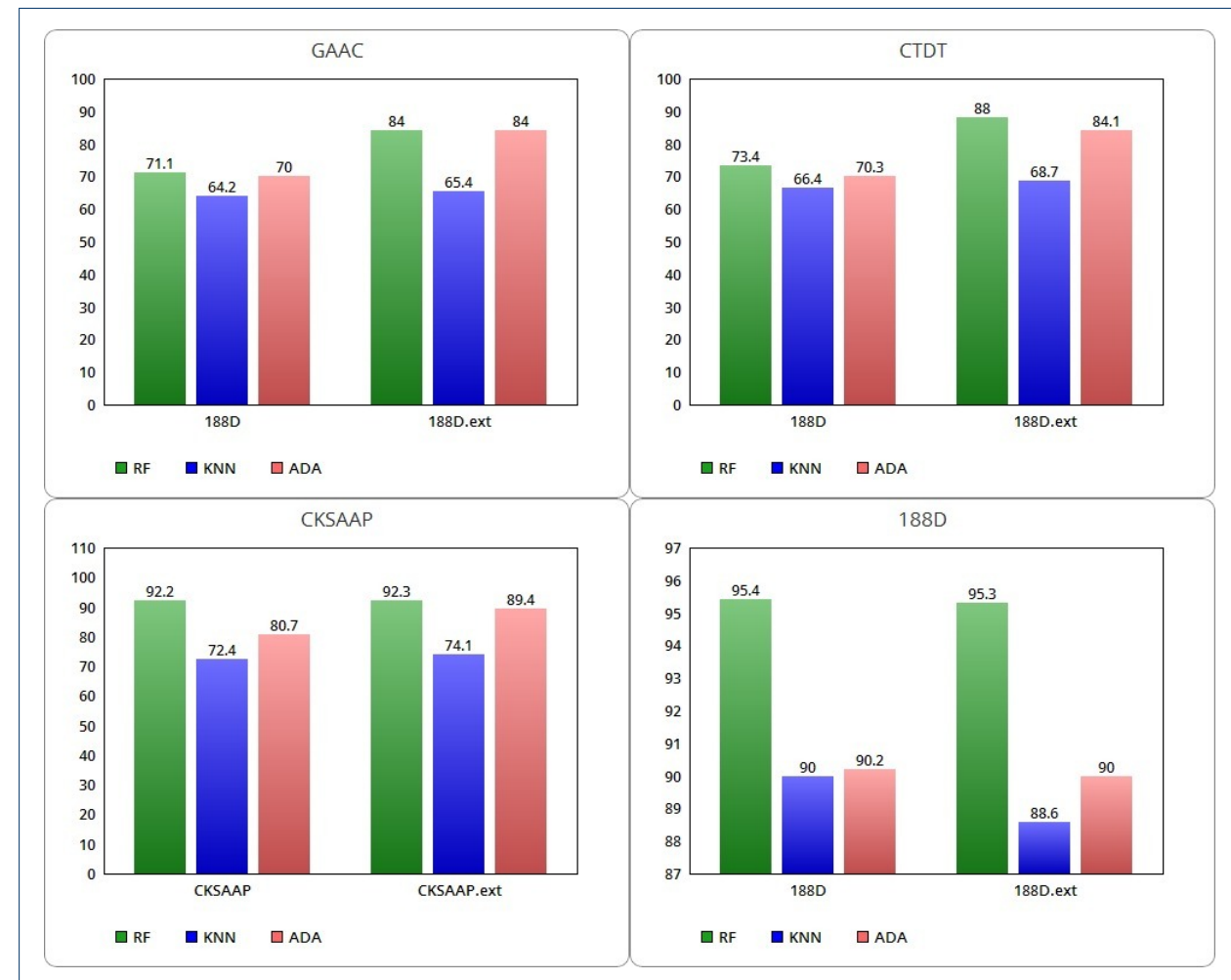

Figure 3 Comparison between GAAC, CTDT, CKSAAP and 188D ACC with related extended classes with SNARE (on D128 dataset). 
Table 8 Performance for average SN and SP on the D128 dataset.

\begin{tabular}{ccccccc}
\hline & \multicolumn{3}{c}{ Sensitivity } & \multicolumn{3}{c}{ Specificity } \\
\hline & RF & KNN & ADA & RF & KNN & ADA \\
\hline GAAC & $80.1 \%$ & $\mathbf{6 5 . 7 \%}$ & $74.5 \%$ & $62.2 \%$ & $63 \%$ & $65.4 \%$ \\
GAAC.ext & $\mathbf{8 4 \%}$ & $62.2 \%$ & $\mathbf{8 8 . 6 \%}$ & $\mathbf{8 3 . 9 \%}$ & $\mathbf{6 9 \%}$ & $\mathbf{7 9 . 2} \%$ \\
\hline CTDT & $74.7 \%$ & $\mathbf{7 0 . 4 \%}$ & $70 \%$ & $72.2 \%$ & $62.3 \%$ & $70.5 \%$ \\
CTDT.ext & $\mathbf{8 7 . 6 \%}$ & $64.7 \%$ & $\mathbf{8 4 . 7 \%}$ & $\mathbf{8 8 . 3 \%}$ & $\mathbf{7 3 \%}$ & $\mathbf{8 3 . 4 \%}$ \\
\hline CKSAAP & $89.7 \%$ & $55.4 \%$ & $80.2 \%$ & $95 \%$ & $89.4 \%$ & $81.3 \%$ \\
CKSAAP.ext & $\mathbf{9 0 . 1 \%}$ & $\mathbf{5 7 \%}$ & $\mathbf{8 9 . 5 \%}$ & $95 \%$ & $\mathbf{9 1 . 2 \%}$ & $\mathbf{8 9 . 4 \%}$ \\
\hline 188D & $\mathbf{9 5 . 7 \%}$ & $\mathbf{8 9 \%}$ & $88.5 \%$ & $95.1 \%$ & $\mathbf{9 1 \%}$ & $\mathbf{9 2 \%}$ \\
188D.ext & $95.5 \%$ & $88 \%$ & $\mathbf{8 8 . 8 \%}$ & $95.1 \%$ & $89.2 \%$ & $91.2 \%$
\end{tabular}

Table 9 Comparision of MCC for the DUNI and D128 datasets.

\begin{tabular}{ccccc}
\hline \multicolumn{4}{c}{ Matthews correlation coefficient } \\
\hline \multirow{2}{*}{ GAAC.ext } & Dataset & MCC RF & MCC KNN & MCC ADA \\
& DUNI & $\mathbf{0 . 7 4}$ & $\mathbf{0 . 7 6}$ & 0.61 \\
& D128 & 0.69 & 0.32 & $\mathbf{0 . 7 0}$ \\
\hline \multirow{2}{*}{ CTDT.ext } & DUNI & 0.77 & $\mathbf{0 . 8 1}$ & 0.49 \\
& D128 & 0.77 & 0.39 & $\mathbf{0 . 7 0}$ \\
\hline \multirow{2}{*}{ CKSAAP.ext } & DUNI & 0.77 & 0.73 & 0.69 \\
& D128 & $\mathbf{0 . 8 6}$ & 0.53 & $\mathbf{0 . 8 0}$ \\
\hline \multirow{2}{*}{ 188D.ext } & DUNI & 0.84 & $\mathbf{0 . 8 7}$ & 0.70 \\
& D128 & $\mathbf{0 . 9 1}$ & 0.81 & $\mathbf{0 . 8 1}$ \\
\hline
\end{tabular}

Comparison between the DUNI and the D128 datasets

Carrying out experiments on unbalanced datasets or balanced datasets affects the automatic learning of the different ML algorithms. In fact, it has been observed that when tests are performed on an unbalanced dataset, greater accuracy is achieved since the classification of each test sample towards the majority class prevails [35]. Consequently, choosing a balanced dataset for training tests can lead to a higher quality of classification predictions. Since the ACC calculation is sensitive to the imbalance class in order to compare the DUNI and D128 datasets, following the SNARER descriptors introduction, we have used the Matthews Correlation Coefficient (MCC) [36]. MCC varies in the range $[-1 ; 1]$ and it is used as a classification quality measure also in cases where the datasets have different sizes. When the MCC value is 1 , it indicates a perfect forecast. If it returns a value of -1 it represents a perfect negative correlation while 0 means that the classifier returns only a forecast no better than a random one.

$$
M C C=\frac{T P \times T N-F P \times F N}{\sqrt{(T P+F P)(T P+F N)(T N+F P)(T N+F N)}}
$$

In Table 9, we present the comparison between the MCC metrics for RF, KNN and ADA trained on the DUNI and D128 datasets with the extended descriptors classes.

The MCC (see Figure 4) of RF improves on the balanced dataset, except for a decrease with the GAAC discriminant features and for no change on the CTDT class. The MCC of KNN is lowered for all combined descriptors, significantly for GAAC, CTDT and CKSAAP. In contrast, ADA's MCC is significantly improved in all four conditions.

\section{Conclusion}

In recent decades, following the exponential increase in data from gene sequencing, it has become necessary to explore different ML techniques for the protein identification, in support of traditional methods [37]. Recent studies on SNARE proteins 


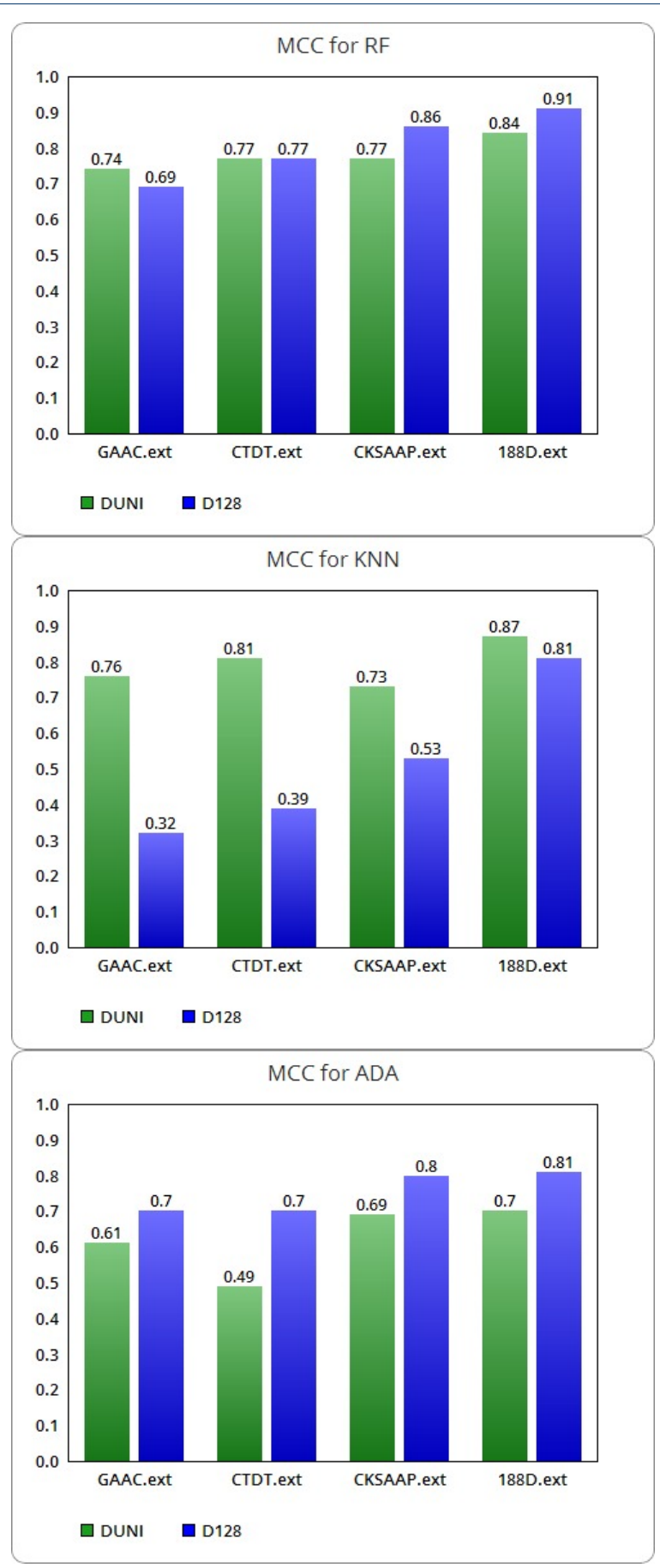

Figure 4 Graphic visualization of MCC for RF,KNN and ADA algorithms.

have shown that their complexes are spoken in the release of neurotransmitters and that their dysfunction is the basis of neurodegenerative, neural developmental and neuropsychiatric disorders. The importance of recognizing them with increasingly precision has a significant biological impact for identifying the aforementioned pathological conditions [38]. The aim of classifying these proteins allows researchers 
to understand the biological pathways in which they are involved and by increasing their knowledge, they can improve the possible therapeutic approach.

In this work, we tested different feature extraction methods on a balanced and an unbalanced dataset, with and without the new contribution of SNARER descriptors addition, in order to examine the role of balanced and unbalanced training in the classification of SNARE binary proteins. Consequently, we compared the behavior of three ML algorithms (RF, KNN and ADA) on the homogeneous and non-homogeneous datasets.

The ML models were evaluated calculating the ACC, SN and SP average values. Our results showed that the performance of the ML algorithms, with the extension of the SNARER descriptors to the feature sets used, improved on both datasets in terms of average ACC. This improvement is greater for RF, KNN and ADA algorithms with the combination of SNARER descriptors to the $188 \mathrm{D}$ class. In particular, our best results on the balanced and non-redundant dataset D128 are $92.3 \%$ of ACC, $90.1 \%$ for SN and $95 \%$ for SP with the RF algorithm and with the extended class CKSAAP.ext. By evaluating the MCC for RF, KNN and ADA on both datasets trained with extended feature sets, the ADA algorithm benefited from better performance applied on the balanced dataset. On the contrary, KNN has worsened in terms of performance, reaching a higher value only for the 188D class. Specifically, the algorithms trained on the balanced dataset produce a better $\mathrm{MCC}$, especially for RF and more for ADA, which recovers both in terms of ACC, $\mathrm{SP}$ and $\mathrm{SN}$ in all the considered tests. KNN, on the contrary, appears to have lower performance in terms of MCC compared to the other algorithms considered.

As future work, it is possible to extend the analysis to also identify the SNARE proteins sub-categories based on their structural features, Q-SNAREs and RSNAREs. Furthermore, it would be useful to explore the use of different classes of descriptors, also combined with each other, which can guarantee a better classification of the proteins under examination.

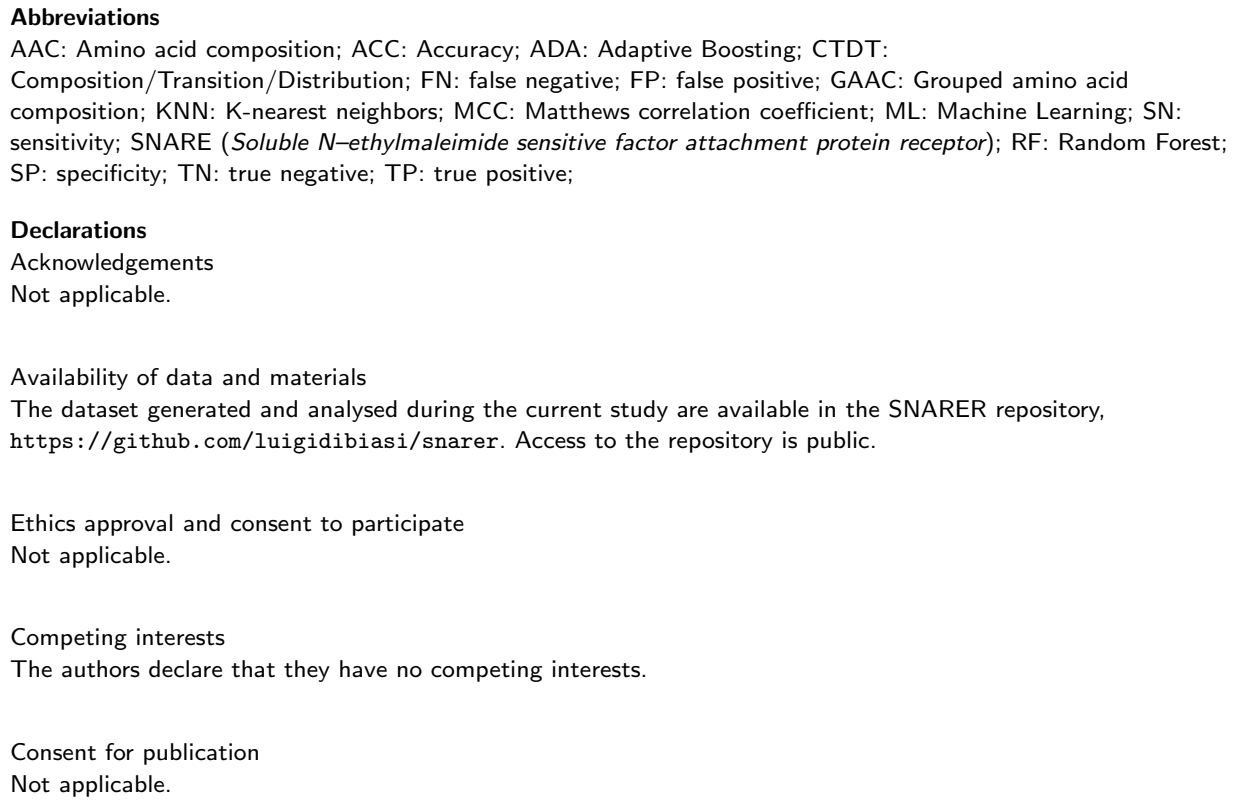


Authors' contributions

LDB designed the algorithm. AAC, MR and GF contributed to writing the manuscript. All authors read and approved the final manuscript.

Funding

Not applicable.

Author details

Department of Computer Science, University of Salerno, Fisciano, IT.

References

1. Ungar, D., Hughson, F.M.: Snare protein structure and function. Annual review of cell and developmental biology 19(1), 493-517 (2003)

2. Chen, Y.A., Scheller, R.H.: Snare-mediated membrane fusion. Nature reviews Molecular cell biology 2(2), 98-106 (2001)

3. Sutton, R.B., Fasshauer, D., Jahn, R., Brunger, A.T.: Crystal structure of a snare complex involved in synaptic exocytosis at 2.4 å resolution. Nature 395(6700), 347-353 (1998)

4. Fasshauer, D., Sutton, R.B., Brunger, A.T., Jahn, R.: Conserved structural features of the synaptic fusion complex: Snare proteins reclassified as q-and r-snares. Proceedings of the national academy of sciences 95(26), 15781-15786 (1998)

5. Ramakrishnan, N.A., Drescher, M.J., Drescher, D.G.: The snare complex in neuronal and sensory cells. Molecular and Cellular Neuroscience 50(1), 58-69 (2012)

6. Yang, X., Kaeser-Woo, Y.J., Pang, Z.P., Xu, W., Südhof, T.C.: Complexin clamps asynchronous release by blocking a secondary ca2+ sensor via its accessory $\alpha$ helix. Neuron 68(5), 907-920 (2010)

7. Guerini, F.R., Bolognesi, E., Chiappedi, M., Manca, S., Ghezzo, A., Agliardi, C., Sotgiu, S., Usai, S., Matteoli, M., Clerici, M.: Snap-25 single nucleotide polymorphisms are associated with hyperactivity in autism spectrum disorders. Pharmacological Research 64(3), 283-288 (2011)

8. Etain, B., Dumaine, A., Mathieu, F., Chevalier, F., Henry, C., Kahn, J., Deshommes, J., Bellivier, F., Leboyer, M., Jamain, S.: A snap25 promoter variant is associated with early-onset bipolar disorder and a high expression level in brain. Molecular Psychiatry 15(7), 748-755 (2010)

9. Nakamura, K., Anitha, A., Yamada, K., Tsujii, M., Iwayama, Y., Hattori, E., Toyota, T., Suda, S., Takei, N., Iwata, Y., et al.: Genetic and expression analyses reveal elevated expression of syntaxin 1a (stx1a) in high functioning autism. International Journal of Neuropsychopharmacology 11(8), 1073-1084 (2008)

10. Garcia-Reitböck, P., Anichtchik, O., Bellucci, A., lovino, M., Ballini, C., Fineberg, E., Ghetti, B., Della Corte, L., Spano, P., Tofaris, G.K., et al.: Snare protein redistribution and synaptic failure in a transgenic mouse model of parkinson's disease. Brain 133(7), 2032-2044 (2010)

11. Smith, R., Klein, P., Koc-Schmitz, Y., Waldvogel, H.J., Faull, R.L., Brundin, P., Plomann, M., Li, J.-Y.: Loss of snap-25 and rabphilin 3a in sensory-motor cortex in huntington's disease. Journal of neurochemistry 103(1), 115-123 (2007)

12. Reuter, J.A., Spacek, D.V., Snyder, M.P.: High-throughput sequencing technologies. Molecular cell 58(4), 586-597 (2015)

13. Meng, J., Wang, J.: Role of snare proteins in tumourigenesis and their potential as targets for novel anti-cancer therapeutics. Biochimica et Biophysica Acta (BBA)-Reviews on Cancer 1856(1), 1-12 (2015)

14. Honer, W.G., Falkai, P., Bayer, T.A., Xie, J., Hu, L., Li, H.-Y., Arango, V., Mann, J.J., Dwork, A.J., Trimble, W.S.: Abnormalities of snare mechanism proteins in anterior frontal cortex in severe mental illness. Cerebral Cortex 12(4), 349-356 (2002)

15. Kloepper, T.H., Kienle, C.N., Fasshauer, D.: An elaborate classification of snare proteins sheds light on the conservation of the eukaryotic endomembrane system. Molecular biology of the cell 18(9), 3463-3471 (2007)

16. Le, N.Q.K., Nguyen, V.-N.: Snare-cnn: a $2 d$ convolutional neural network architecture to identify snare proteins from high-throughput sequencing data. PeerJ Computer Science 5, 177 (2019)

17. Li, G.: Identification of snare proteins through a novel hybrid model. IEEE Access 8, 117877-117887 (2020)

18. Consortium, G.O.: Gene ontology consortium: going forward. Nucleic acids research 43(D1), 1049-1056 (2015)

19. Li, W., Godzik, A.: Cd-hit: a fast program for clustering and comparing large sets of protein or nucleotide sequences. Bioinformatics 22(13), 1658-1659 (2006)

20. Orengo, C.A., Jones, D.T., Thornton, J.M.: Protein superfamilles and domain superfolds. Nature 372(6507), 631-634 (1994)

21. Ong, S.A., Lin, H.H., Chen, Y.Z., Li, Z.R., Cao, Z.: Efficacy of different protein descriptors in predicting protein functional families. Bmc Bioinformatics 8(1), 300 (2007)

22. Patil, K., Chouhan, U.: Relevance of machine learning techniques and various protein features in protein fold classification: A review. Current Bioinformatics 14(8), 688-697 (2019)

23. Luo, R.-y., Feng, Z.-p., Liu, J.-k.: Prediction of protein structural class by amino acid and polypeptide composition. European Journal of Biochemistry 269(17), 4219-4225 (2002)

24. Chou, K.-C.: Prediction of protein cellular attributes using pseudo-amino acid composition. Proteins: Structure, Function, and Bioinformatics 43(3), 246-255 (2001)

25. Chen, Z., Zhao, P., Li, F., Leier, A., Marquez-Lago, T.T., Wang, Y., Webb, G.I., Smith, A.I., Daly, R.J., Chou, K.-C., et al.: ifeature: a python package and web server for features extraction and selection from protein and peptide sequences. Bioinformatics 34(14), 2499-2502 (2018)

26. Chen, K., Kurgan, L.A., Ruan, J.: Prediction of flexible/rigid regions from protein sequences using k-spaced amino acid pairs. BMC structural biology 7(1), 25 (2007)

27. Cai, C., Han, L., Ji, Z.L., Chen, X., Chen, Y.Z.: Svm-prot: web-based support vector machine software for functional classification of a protein from its primary sequence. Nucleic acids research 31(13), 3692-3697 (2003) 
28. Kawashima, S., Kanehisa, M.: Aaindex: amino acid index database. Nucleic acids research 28(1), 374-374 (2000)

29. FAUCHÈRE, J.-L., Charton, M., Kier, L.B., Verloop, A., Pliska, V.: Amino acid side chain parameters for correlation studies in biology and pharmacology. International journal of peptide and protein research 32(4), 269-278 (1988)

30. Freund, Y., Schapire, R.E.: A decision-theoretic generalization of on-line learning and an application to boosting. Journal of computer and system sciences 55(1), 119-139 (1997)

31. Wu, X., Kumar, V., Quinlan, J.R., Ghosh, J., Yang, Q., Motoda, H., McLachlan, G.J., Ng, A., Liu, B., Philip, S.Y., et al.: Top 10 algorithms in data mining. Knowledge and information systems 14(1), 1-37 (2008)

32. Ho, T.K.: Random decision forests. In: Proceedings of 3rd International Conference on Document Analysis and Recognition, vol. 1, pp. 278-282 (1995). IEEE

33. WEKA, S.: The waikato environment for knowledge analysis. University of Waikato, Hamilton, New Zealand: University of Waikato (1995)

34. Piotto, S., Di Biasi, L., Concilio, S., Castiglione, A., Cattaneo, G.: Grimd: distributed computing for chemists and biologists. Bioinformation 10(1), 43 (2014)

35. Wei, Q., Dunbrack Jr, R.L.: The role of balanced training and testing data sets for binary classifiers in bioinformatics. PloS one 8(7), 67863 (2013)

36. Boughorbel, S., Jarray, F., El-Anbari, M.: Optimal classifier for imbalanced data using matthews correlation coefficient metric. PloS one 12(6), 0177678 (2017)

37. Gevaert, K., Vandekerckhove, J.: Protein identification methods in proteomics. ELECTROPHORESIS: An International Journal 21(6), 1145-1154 (2000)

38. Chen, F., Chen, H., Chen, Y., Wei, W., Sun, Y., Zhang, L., Cui, L., Wang, Y.: Dysfunction of the snare complex in neurological and psychiatric disorders. Pharmacological Research, 105469 (2021) 